\title{
Impact Damage Detection in Fiberglass Composites Using Low Acoustic Impedance-Based PZT Transducers
}

\author{
Ali Mohammadabadi ${ }^{1,2}$, Alireza Chamani ${ }^{3}$, Roberto Dugnani $^{2, *}$ \\ ${ }^{1}$ Department of Mechanical Engineering, University of Michigan - Shanghai Jiao Tong University Joint Institute, Shanghai, China \\ ${ }^{2}$ Department of Mechanical Engineering, University of Maryland, Baltimore County, MD 21250 USA \\ ${ }^{3}$ Department of Mechanical Engineering, Johns Hopkins University, Baltimore County, MD 21218 USA \\ Received 23 March 2019; received in revised form 04 May 2019; accepted 20 July 2019
}

\begin{abstract}
The objective of this work is to evaluate the feasibility of detecting damages caused by impacts in fiberglass-epoxy composites using lead-zirconate-titanate (PZT) transducers. Impacts were created by a hammer (unquantified energy) and an automatic impact system (quantified energy) in multiple composite sheets. The mechanism of the damage detection relies on the impedance measurement by a low acoustic impedance (LAI) transducer, which resonates in the radial mode rather than the regular thickness mode. The effect of surface roughness was investigated by using specimens with different quantified surface roughness values. It was shown that the final results are heavily affected by the rough surface and hard boundary conditions. Mainly, we aimed to evaluate the efficiency of low acoustic impedance technique in the damage detection of composites. The tests were carried out in the energy ranges of 10 to 54 joules. The surface of each specimen was gridded with a step size of 5 $\mathrm{mm}$, and the impedance was measured for each location. The final results were normalized using a no-load condition as the reference point. It was shown that the proposed portable and easy-to-use LAI setup could detect the damages qualitatively. The normalized measured impedance was variable, but it showed a significant increase, in some cases as high as $100 \%$, on the impact's region.
\end{abstract}

Keywords: robotics, IoT, internet, teleoperation, robot remote control, delay time, virtual private network, VPN

\section{Introduction}

Composite structures are known for being susceptible to manufacturing defects and in-service damage. Since defects in composites are not always visually apparent, Non-Destructive Techniques (NDT) are often employed to detect and quantify damages [1-2]. Composite materials such as carbon-epoxy and fiberglass-epoxy plates are frequently used as structural elements in both the civil and aerospace industries. Composite materials have numerous applications in the biomedical field as well. For example, Monich et al. [3] reviewed the properties of PEEK composites for biomedical applications. Since the applications for composite materials are steadily growing, demands for affordable and reliable inspection techniques for early detection of damages are increasing. Structural Health Monitoring (SHM) provides a damage detection and interpretation procedure that will lead to a higher degree of reliability and safety, and lower inspection and maintenance costs. Generally, damages in composites appear in the shape of surface, transverse, and micro-cracks and also, delamination of layers, as discussed by Naebe et al. [4]. The focus of this study is to evaluate damage in fiberglass composites, which are low-cost and show good chemical properties for various applications. Different sources of damage, including environmental conditions, impacts, and loads, have been selected for this group of composites and introduced by Croitoru et al. [5].

* Corresponding author. E-mail address: roberto.dugnani@ sjtu.edu.cn Tel: +86-21-34206765 Ext. 5181 
It has been shown that damages caused by the impact can severely decrease the durability of the structures and lead to risky and costly failures, as reviewed by Agrawal et al. [6]. To avoid these failures, various NDT techniques have been employed. Previous studies evaluated different techniques to localize and quantify the damages in composites. For example, ultrasound scanning, thermographic inspection, PZT-based, and the vibration-based inspection have been used and compared to detect the damages in composite structures [7-8]. Another study by Maierhofer et al. [9] investigated the thermographic testing to evaluate damages in carbon fiber reinforced polymer composites. Other techniques, such as vibration methods [10] and radiography [11] have been used to detect the damages in composite structures. Even though these techniques are capable of providing various quantitative results regarding the damage, they are all relatively slow and expensive processes. In several applications, it is more cost- and time-efficient to have a fast and qualitative technique for the primary evaluation of the structures based on the daily routines. In this study, we discuss the LAI technique, which can be used to detect near-surface damages in fiberglass-epoxy composites.

In general, PZT transducers have been used for ultrasound testing (UT) and PZT-based inspection techniques already [12-14]. Although these PZT transducers have other capabilities such as energy harvesting as studied by Nezami et al. [15], their main application remains in the field of SHM and NDT. In the case of SHM applications, these PZT transducers are mostly embedded in or bonded to the host structures to monitor the mechanical impedance signatures. Recent studies provided more features to identify and classify the conditions of damages. For example, using damage indices obtained from Gaussian confidence ellipses of electrical admittance could improve the identification and analysis of damages [16]. In addition to the expensive testing equipment and required expertise to analyze the data, acoustic techniques such as UT and PZT-based methods have a limitation in detecting the near-surface defects, including the delamination of composites [17-18]. In the case of near-surface defects, the echo lies within a wavelength of the propagated wave and cannot be detected properly [19]. Using the impedance signature of the structures is not a new idea, but they have only been used in a regular thickness mode of the PZT transducer [20-22]. The sensitivity of the impedance-based technique is related to several factors, for example, the coupling interfaces [23]. We have shown that the radial mode resonance provides several advantages for SHM and NDT applications [19, 24-25]. Briefly, in the radial resonance mode, we use the concept of self-sensing in PZT transducers, which was discussed in detail by Dugnani [24]. The operational frequency of the thickness mode transducers is much higher than the radial ones. Therefore the characteristic acoustic impedance of the thickness mode is one order of magnitude higher than the fluids and polymer materials. To reduce this impedance mismatch, several intermediate matching layers are required to ensure the efficient delivery of acoustic energy. The proposed impedance-based technique should not be confused or equated with the regular electromechanical impedance (EMI) method.

The EMI method has been utilized for the last 2 decades by coupling (i.e., physically bonding) transducers to a structure and using them to analyze the change in the resonant behavior of the structure (i.e., modal analysis). Examples of this use of the EMI method can be found in the classic work of Liang et al. [26], which described the behavior of PZT transducers physically bonded to a beam. In particular, Liang et al.'s approach monitored changes in the resonant behavior of the entire beam (i.e., variable monitored is the "structural stiffness" or the "resonant frequency" of the beam). In terms of hardware, the EMI method referred by the reviewer utilizes transducer(s) which are physically bonded to the structure; its working principles relies on comparing the modal response of the damaged and undamaged structure. Furthermore, the wavelength excited by the classic EMI method is normally quite long since a large structure needs to be monitored (i.e., in the case of the beam, the wavelength is comparable to the beam's length).

The proposed impedance-based technique is fundamentally different both in the hardware (the transducer is not physically bonded to the structure and could be actuated in either radial or -more often- in the thickness mode), the nature of the measurements (in our case the local, complex acoustic impedance is tracked), and the wavelengths utilized (the wavelength must be comparable to the thickness, not the length of the structure). In the method presented in our paper, the structure only 
locally interacts with the transducer by affecting the acoustic signal's transmission and damping characteristics (the structure, which is locally investigated through its thickness, behaves like a "transmission line" or an "acoustic filter"). Unlike the classic EMI methods, our transducer can be positioned at any location on a structure to investigate the through-the-thickness response at such location (i.e., a local debond essentially acts either like a shorter transmission line or a lossier transmission line for highly damaged matrix). One huge advantage of our method over classic EMI methods is that, since the structure is not excited as a whole, there are no "nodal" locations which could not be investigated by the transducer. Finally, the reason why both we and Liang et al. use an impedance analyzer is because both the large-scale modal response of a structure and the local acoustic impedance of a transmission line in series with our transducer are complex signals comprised of a phase and a magnitude (commonly referred to by the generic term "impedance").

\section{Experimental Study}

\subsection{LAI PZT transducer}

The effective acoustic impedance of PZT transducer for the radial resonance mode is lower than the thickness mode, Fig. 1 , and changing the aspect ratio of the diameter and the thickness, in the case of the cylindrical transducer, will help to tune the required acoustic impedance [24]. It is shown in Fig. 1 that the maximum dissipated energy by the transducer corresponds with the minimum real part value of the admittance. Therefore, the maximum energy can be transmitted to the structure for the radial resonance in lower acoustic impedances. This feature provides an advantage for LAI materials such as polymer composites by eliminating the need for the matching layers. We verified the validity of this approach in the detection of debonding in polymer materials [27] and also a primary experiment with the composite materials [25]. The current study investigates the detection of damages in composites more precisely.

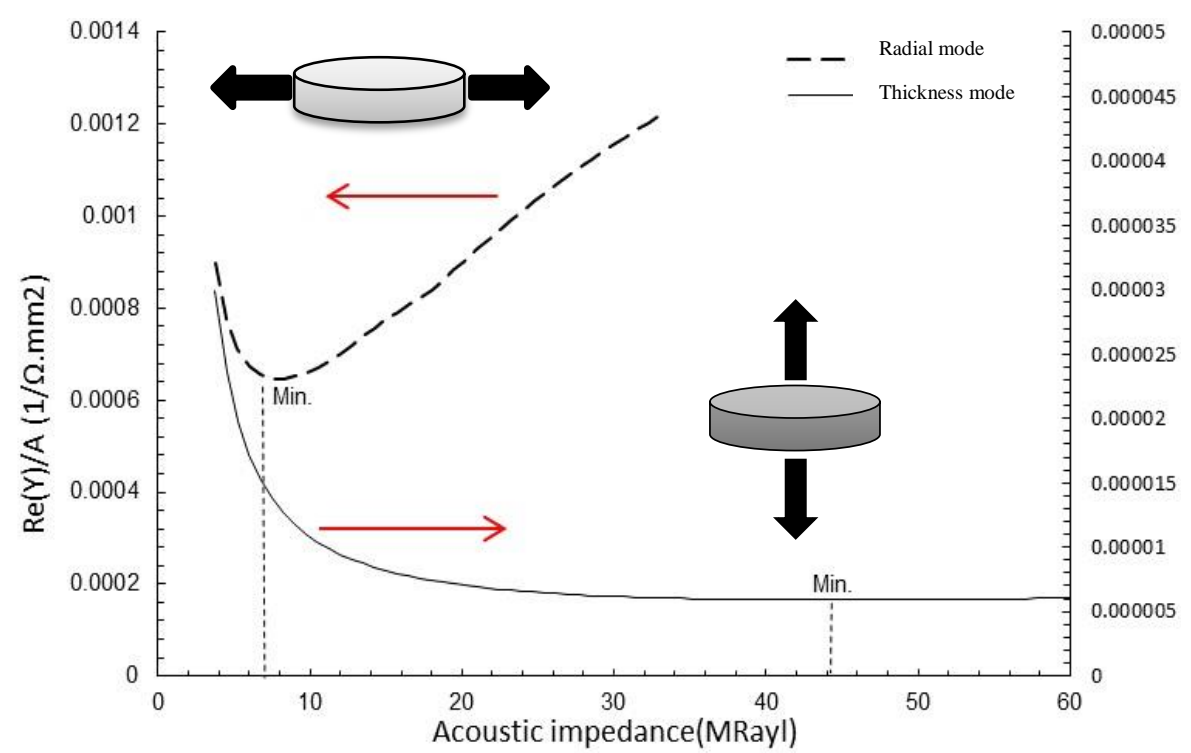

Fig. 1 Real part of admittance for the radial and thickness modes [27]

\subsection{Test setup}

A ceramic PZT transducer, PZT-5A, with the resonance frequency of $245 \mathrm{kHz}$, diameter of $10 \mathrm{~mm}$ and thickness of 3.7 mm was provided by APC International Ltd (American Piezo Ceramics Ltd). To analyze the impedance, an evaluation board, AD 5933, was provided by the Analog Devices Co. We designed and printed a frame for the experiment [27]. The schematic view of the transducer, experiment setup, and the final fabricated transducer are shown in Fig. 2.

We then glued the PZT transducer to a flexible copper clad laminate using a conductive glue and soldered the terminals to a coaxial cable. The cable was connected to the input sockets of the evaluation board, and the system was calibrated by a $50 \Omega$ resistor. The frequency range was adjusted such that the resonance frequency falls on the mid-range. To have a reference point 
to normalize the measured impedance, the PZT transducer was excited in the air without any loading. The PZT transducer was placed on the composite specimen and coupled by ultrasound gel. For each location, the acoustic impedance was measured and stored. The collection of data was processed and plotted in Microsoft Excel or MATLAB.

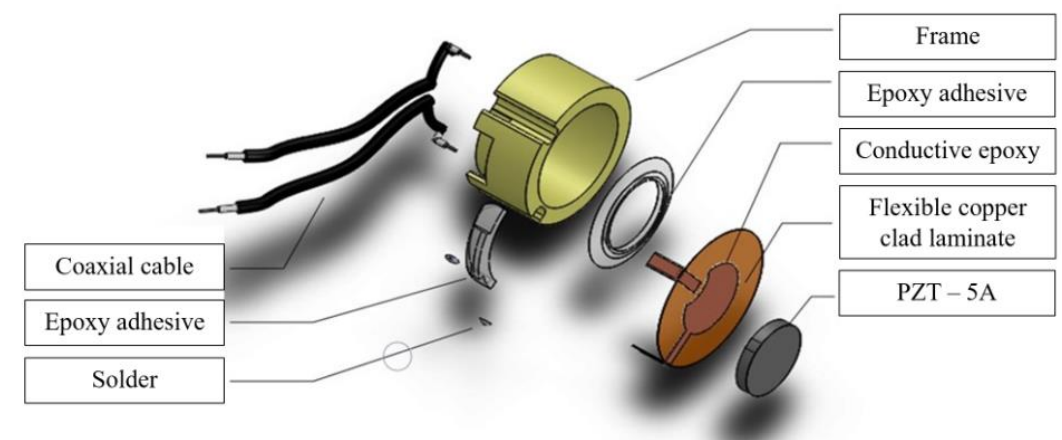

(a) Exploded view of custom PZT transducer

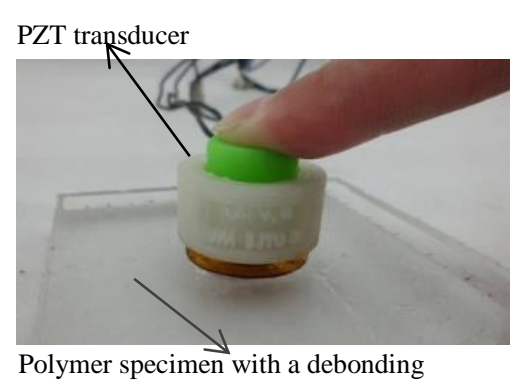

(b) Transducer's prototype

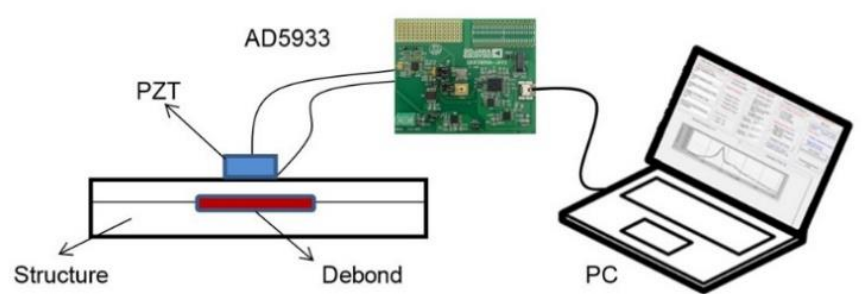

(c) Setup schematic

Fig. 2 Device and test setup [27]

The AD5933 program provided three types of measurements that can be used to study the sensitivity of the setup. These three measuring parameters were impedance's magnitude, frequency, and impedance' phase. The impedance method has been tested in several experiments and is the main idea of this study [24, 25, 27]. However, the frequency shifting and even the phase change could detect the presence of defects, but they were not as certain as the magnitude of the impedance. Theoretically, it was shown that the resonant frequency would not change significantly [19, 24].

\subsection{The effect of surface roughness}

Optimal coupling between the PZT transducer and the specimen plays a critical role in PZT related techniques [28]. Surface conditions may change due to the natural factors like corrosion or operational factors like damages by impacts and external forces. In the case of very rough surfaces, trapped airs between the transducer and specimen reduce the efficacy of the technique. To study the effect of surface roughness, a Polymethylmethacrylate (PMMA) disk was cut with a diameter of 100 $\mathrm{mm}$ and a thickness of $17 \mathrm{~mm}$. One side of the disk was sanded in half with very rough and another half with semi-rough sandpapers. The PMMA disk does not have a reflective surface. Therefore it needed to be coated by a reflective material so that the optical profilometer could measure the roughness of the surface. The disk was then baked in the oven for 6 hours in $50{ }^{\circ} \mathrm{C}$ and then placed in a vacuum chamber to remove all the trapped air. Then the specimen was coated by gold ion sputtering (model E1045 manufactured by Hitachi Co). The estimated thickness of the gold coating layer is as small as $5 \mathrm{~nm}$, to make sure that the coating has minimum interference with the roughness of the surface. Both sides of the disk were coated as the profilometer needed to measure the roughness of both sides. The optical profilometer WYKO NT1100, manufactured by Veeco Co., has been used for measuring the roughness which adjusted the profilometry's window automatically. The average roughness of the surface was measured, and Fig. 3 shows the optical images of three surfaces with different roughness.

The profilometer device provided three average values of roughness, which are Ra (average roughness), Rq (root mean square roughness) and Rt (Vertical distance between the highest peak and lowest valley). These measured values are given in Table 1. 


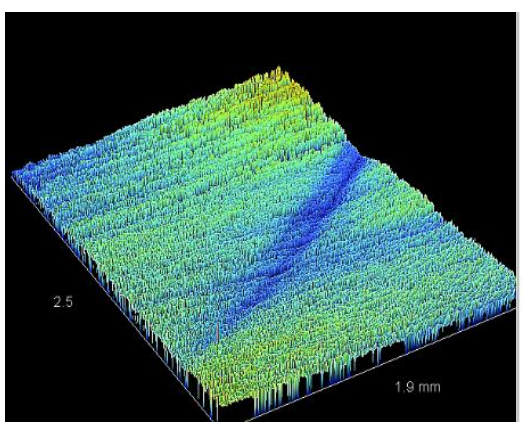

(a) Smooth surface

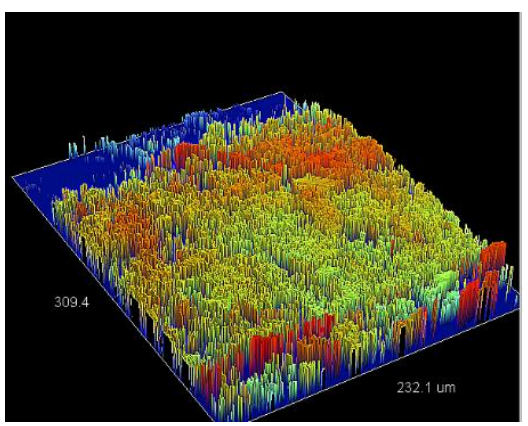

(b) Semi-rough surface

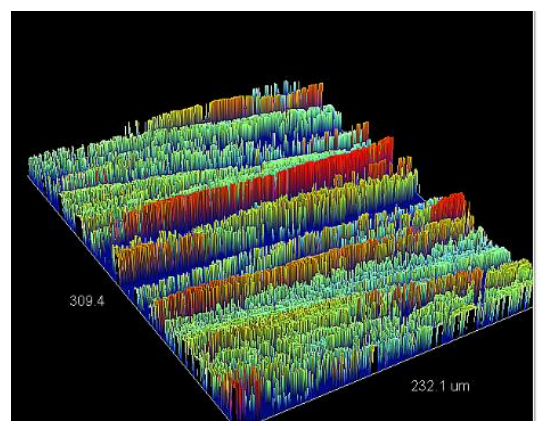

(c) Rough surface

Fig. 3 Optical profiler images of surfaces with different roughness

Table 1 Measured roughness values

\begin{tabular}{|c|c|c|c|}
\hline & $\mathrm{Ra}(\mu \mathrm{m})$ & $\mathrm{Rq}(\mu \mathrm{m})$ & $\mathrm{Rt}(\mu \mathrm{m})$ \\
\hline Smooth & 0.18 & 0.22 & 0.95 \\
\hline Semi- rough & 4.71 & 5.11 & 14.49 \\
\hline Rough & 15 & 15.91 & 33.88 \\
\hline
\end{tabular}

The impedance was measured with two different boundary conditions. First, the disk was placed on an empty paper box which simulates the air beneath of disk. Second, the disk was placed on a thick wooden table to simulate a solid boundary condition. Both sides of the disk were gridded, and the impedance was measured at all points. The transducer was placed on each section of the surface with different roughness values. An adequate amount of gel was employed to ensure the proper coupling between the transducer and the specimen. The real part of impedance was measured for different locations by manually moving of the transducer. To normalize these results, the impedance was measured for a case of the unloaded transducer, means not in touch with the specimen and just free vibrating without any load. Then the measured results from the specimen were divided by the unloaded case results. The average value of the measured impedance was then calculated and normalized using the free resonance value of the PZT transducer. In the case of the hard-solid boundary, the average measured impedance increased around $70 \%$ from smooth to rough surface, but the difference from smooth to the semi-rough surface was as small as $5 \%$. In the case of the soft boundary, the air beneath, the measured average impedance increased around $25 \%$ from the smooth to the rough surface and almost $2 \%$ from the smooth to the semi-rough surface. The results show that in addition to the surface roughness, the boundary conditions affect the measured impedance as well. The normalized impedances are shown in Fig. 4.

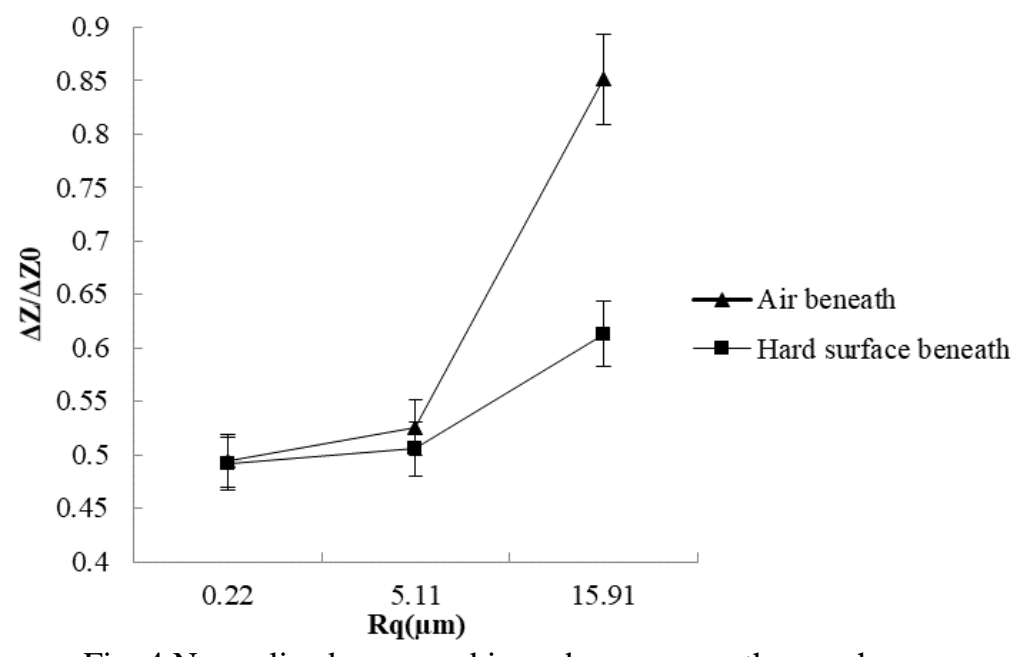

Fig. 4 Normalized measured impedance versus the roughness

\subsection{Composite test specimens}

A set of experiments was carried out to evaluate the efficacy of the impedance-based technique in the detection of impact damages in fiberglass/epoxy composite plates. The composite plates that were used are FR4 Epoxy-Glass sheets, manufactured 
by Leadwin Co, (FR stands for the flame retardant). FR4 epoxy glass composites are alkali-free glass woven fabric base saturated in epoxy resin binder with a 0/90-degrees layout. FR4 composites have high mechanical strength, dielectric properties, and are resistant to heat and humidity. These composites have several versatile applications such as insulating structural parts in electrical equipment. They are also used in the manufacturing of switches, standoffs, washers, arc shields, relays, transformers, and screw strips. The frequency range for both unquantified and quantified impact energies was $230 \sim 260$

$\mathrm{kHz}$. Based on the dimension and characteristics of the employed PZT-5A, the first radial resonance mode appeared within this range. The reference impedance $\Delta \mathrm{Z}_{0}$ was variable for different frequencies in the range, but for a resonance frequency of $245 \mathrm{kHZ}, \Delta \mathrm{Z}_{0}$ was measured as $44021 \Omega$.

\subsubsection{Damage with the unquantified impact energy}

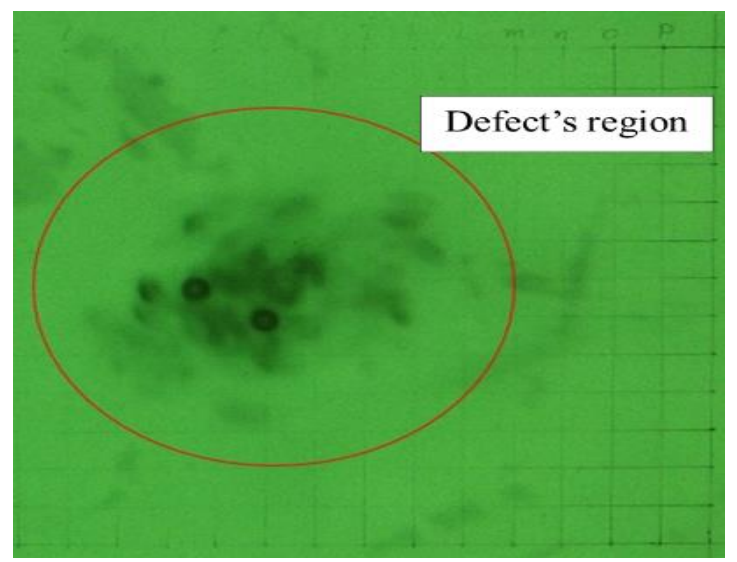

(a) Impact's region

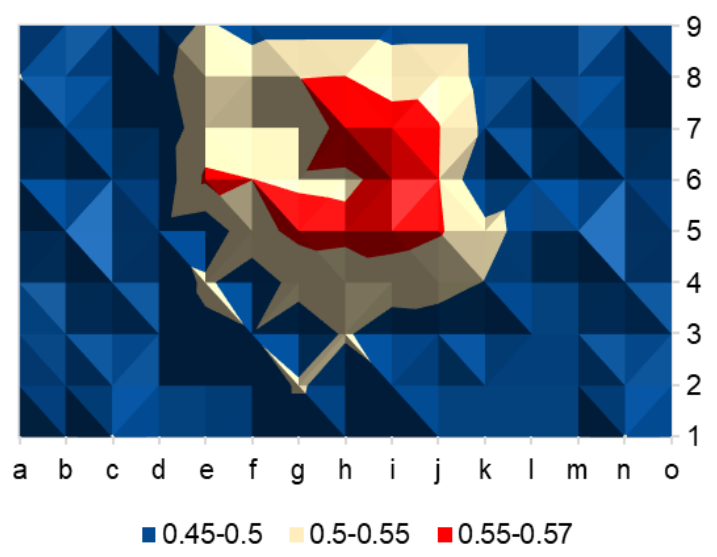

(c) Contour surface plot of the measured impedance

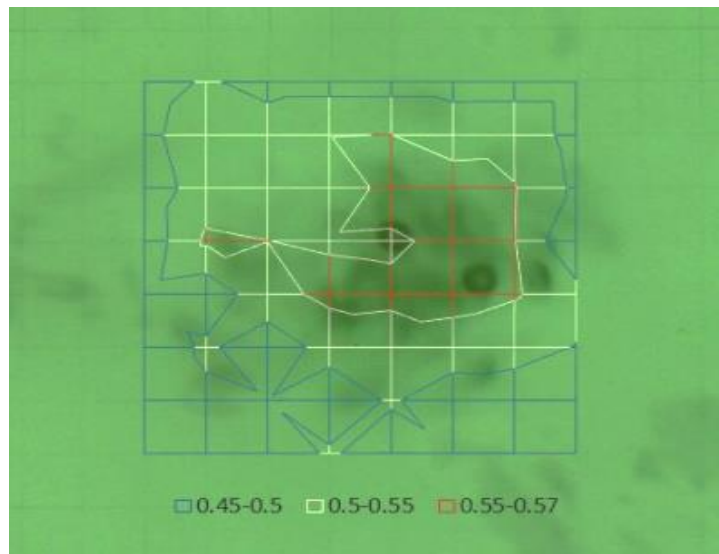

(b) Superimposition of measured impedance and impact's region

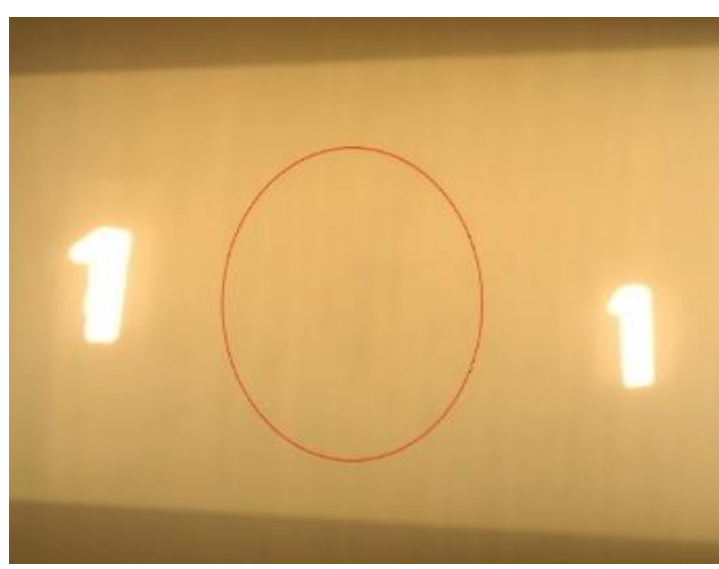

(d) x-ray test image

Fig. 5 Hammer impact's characterization

Impact tests were carried out on FR4 plates with a length of $200 \mathrm{~mm}$, the width of $180 \mathrm{~mm}$ and a thickness of $3 \mathrm{~mm}$. The defect was created by the hammering of the plate. As a preliminary experiment, we did not measure the energy of impact, but the damage was clearly visible in the plate. The surface of the plate was gridded in $10 \times 10 \mathrm{~mm}$ squares. The PZT transducer was then coupled to the surface of the plate by the ultrasound transmission gel manufactured by Mibo Co. The PZT transducer was not affected by the impact, and it was placed and moved over the surface after the impact. The coupling between the transducer and the specimen was ensured by adding adequate gel and manual pushing the transducer over the surface. We added a brief explanation in the text. The schematic view of the defect is shown in Fig. 5(a). The peak value of the impedance magnitude was recorded at each location on the grid. The resonance impedance values were normalized using the free excitation value of the PZT transducer in the air $\left(\Delta \mathrm{Z}_{0}\right)$. The contour surface plot of data is shown in Fig. 5(c). The analyzed data shows that the normalized impedance changes up to $25 \%$ when the PZT transducer reaches the damaged region. The non-damaged region showed a normalized impedance $\left(\Delta \mathrm{Z} / \Delta \mathrm{Z}_{0}\right)$ range of $0.45-0.5$, but when the PZT transducer passed the 
defective region, the normalized impedance increases to $0.5-0.55$. Interestingly, we could also observe several ranges of normalized impedance in the defective region. The visual evaluation of the specimen showed that some regions of the specimen might receive higher impacts and the normalized impedance increased to the $0.55-0.57$ range inside the region of damage, which could be an indicator of the higher intensity of impact. The superimposition of measured impedance and the region of impact is shown in Fig. 5(b). The damage did not have a symmetric shape, but it was contained in a square of $70 \times 70$ $\mathrm{mm}$.

The same composite plate was tested by the X-ray technique, XY-2515 flaw detector manufactured by Liaoning Instrument Co. The specimen was under radiation for 210 seconds. The maximum applied voltage across the $\mathrm{x}$-ray tube was $250 \mathrm{kVP}$ (peak kilovoltage) with a $15 \mathrm{~mA}$ current. The size of the focal spot is $44 \mathrm{~mm}$ with a radiation angle of $40 \mathrm{o}$ and a focus film distance (FFD) of $600 \mathrm{~mm}$. The resulting x-ray image is shown in Fig. 5(d) with the region of defect circled for clarification. The X-ray test by XY-2515 could not detect the impact's damage on the composite plate.

\subsubsection{Damage with the quantified impact energy}

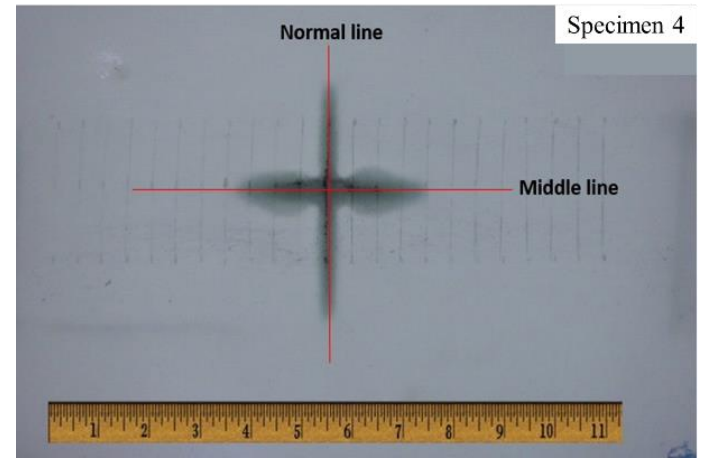

(a) Middle /Normal lines of measurement

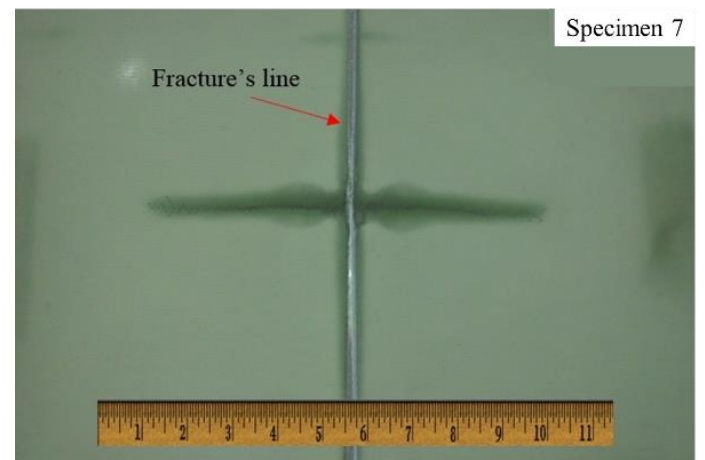

(b) Fractured composite, $\mathrm{E}=54$ joules

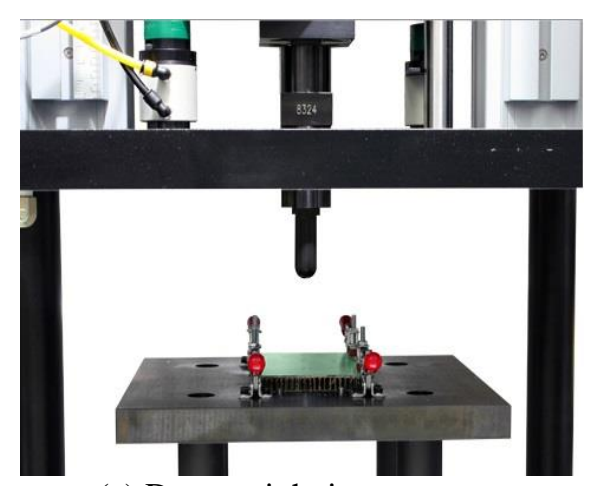

(c) Drop weight impact tester

Fig. 6 Impact test and measurement setup

Seven samples of FR4 fiberglass - epoxy composites were cut in size of $150 \times 100 \times 35 \mathrm{~mm}$. The setup and process of testing were arranged based on Boeing BSS 7260 Type II, class 2, ASTM D7136/D7136M-05 standards which cover measuring of the damage resistance of fiber reinforced polymer matrix composite plates that have been subjected to a drop weight impact test. The CEAST 9350 impact system manufactured by Instron co. was used in this experiment, Fig. 6(c). The testing device was a floor standing impact machine with the impact's energy range of $0.59-757$ joules. The specimen was fixed in four corners with simple supports such that the test weight hits the specimen in the center. The dropping weight simulates an impact on the composite specimen, and it was expected that higher impact's energy could create more severe damages which were larger in size as well. However, it was also expected that the energy of the impact would reach a threshold which would lead to the fracture of the composite's specimen. We started with the impact energy of 10 joules because the damage size was small for energies lower than this, and we were interested in having a large enough damage to test our proposed setup properly. 
We tested the impact energy for a range of 10 to 54 joules, and Table 2 shows the impact data for each specimen. Although the impact's energy is the dominant factor regarding the impact's damage but based on the previous studies [29], the different mass and speed of impactor with the same impact's energy may change the shape of damages. The same phenomenon was observed for the specimens no. 3, 4, and 5, which had the same impact's energy, but the shapes of damage were not identical.

Table 2 Impact's specifications

\begin{tabular}{|c|c|c|c|c|}
\hline Number & Energy $(\mathrm{J})$ & Velocity $(\mathrm{m} / \mathrm{s})$ & Impactor diameter $(\mathrm{mm})$ & Impactor mass $(\mathrm{kg})$ \\
\hline 1 & $\sim 10$ & 1.41 & 26 & 10.131 \\
\hline 2 & $\sim 20$ & 1.98 & 26 & 10.131 \\
\hline 3 & $\sim 30$ & 3.73 & 12.6 & 4.277 \\
\hline 4 & $\sim 30$ & 3.73 & 26 & 4.277 \\
\hline 5 & $\sim 30$ & 3.35 & 26 & 5.277 \\
\hline 6 & $\sim 34$ & 2.59 & 26 & 10.131 \\
\hline 7 & $\sim 54$ & 3.26 & 26 & 10.131 \\
\hline
\end{tabular}

Following the impact, the PZT transducer was placed on the surface of the composite specimen, and the impedance was measured at each point of the grid on the gridded specimen with the step size of $5 \mathrm{~mm}$. The impedance measurement was done for middle and normal lines of testing, as shown in Fig. 6(a). Also, the impedance has been measured over the surface of the plate, and the results were introduced in the form of surface contour plots. The PZT transducer was placed on the surface after the impact, and the impedance was measured for each location of the gridded surface by manually moving of the transducer while the coupling was ensured by adding the adequate gel. The normalized impedance and the frequency plots for the specimen no. 5 are shown in Fig. 7 as an example. The center region of the impact showed a higher normalized impedance, 0.6-0.8, the surrounding region showed a lower impedance, $0.4-0.6$ and the healthy regions of the specimen showed the reference measured impedance 0.2-0.4, Fig. 7(a). The frequency plot showed similar regions approximately, but there were some regions like row 2 in the frequency plot, Fig. 7(b), which the source of elevated frequency was unknown. Therefore, we preferred to use the impedance plots for our purpose.

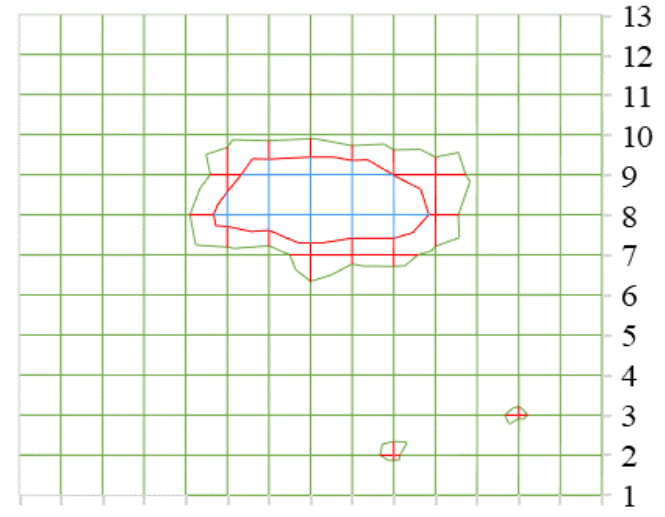

a b c d e f g h i j k 1 m n o

$\square$ 0.2-0.4 $\square 0.4-0.6 \quad \square 0.6-0.8$

(a) Impedance surface plot $\left(\mathrm{Z} / \mathrm{Z}_{0}\right)$

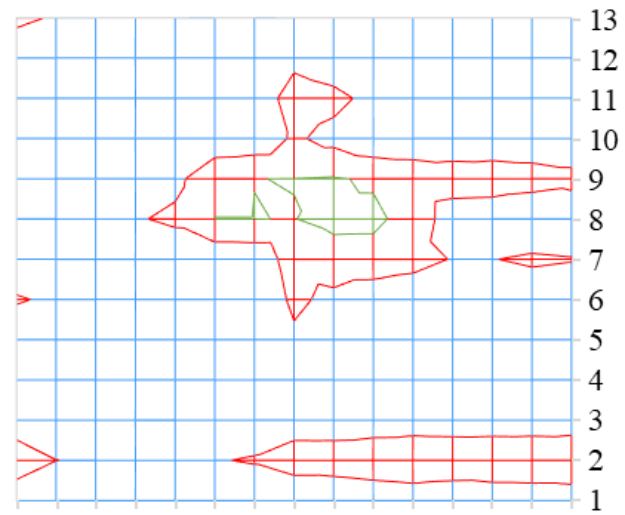

a b c d e f g h i j

$\square 0.98-1 \quad \square 1-1.02 \quad \square 1.02-1.04$

(b) Frequency surface plot $\left(\mathrm{f} / \mathrm{f}_{0}\right)$

Fig. 7 Normalized measured results, specimen no. 5

\section{Results and Discussions}

We tested seven plates with different impact energies. The impact threshold for the fracture was found at 54 joules on the specimen no. 7, which is shown in Fig. 6(b). Since the damage shape is almost symmetric, we decided to measure the impedance over the normal and middle lines with the same length and step size. Then regarding the center of impact, both measurements were averaged corresponding to their similar distance to the center of the impact. Using this technique, we could combine both measurements on a graph with the standard deviation bars. 


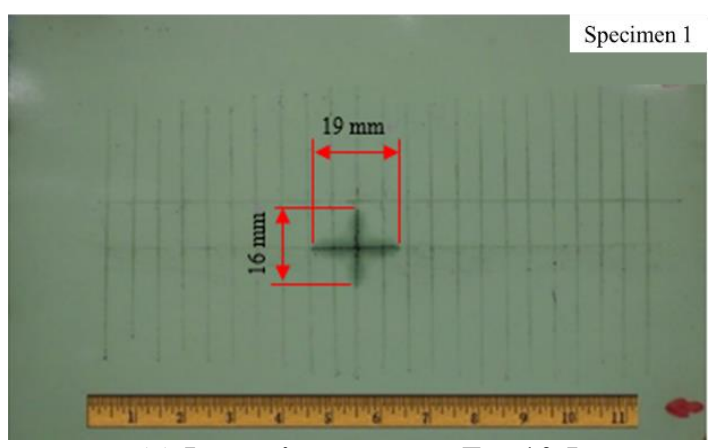

(a) Impact's geometry, $\mathrm{E}=10 \mathrm{~J}$

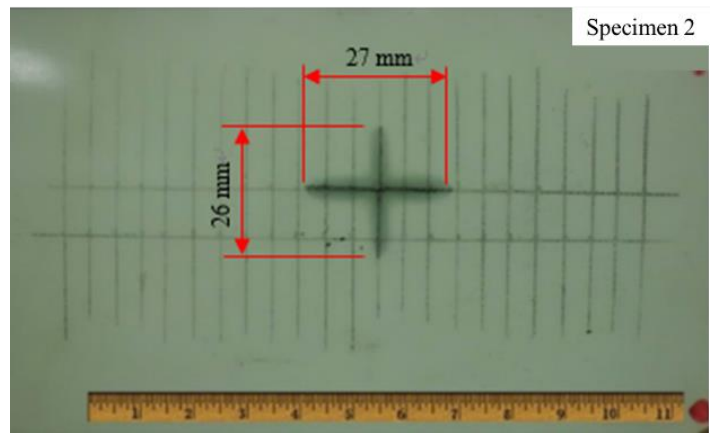

(c) Impact's geometry, $\mathrm{E}=20 \mathrm{~J}$

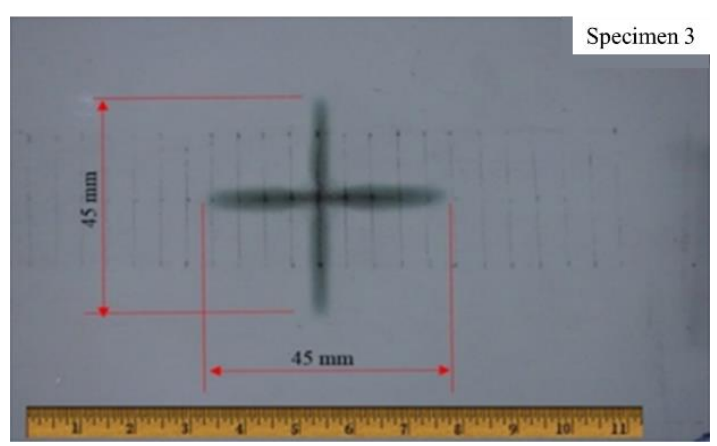

(e) Impact's geometry, $\mathrm{E}=30 \mathrm{~J}$

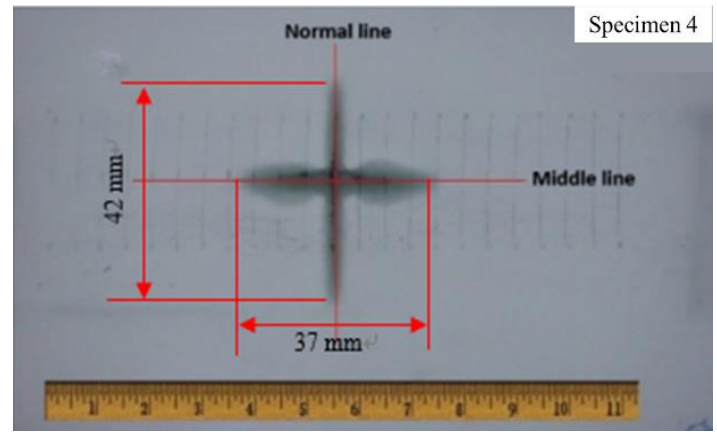

(g) Impact's geometry, $\mathrm{E}=30 \mathrm{~J}$

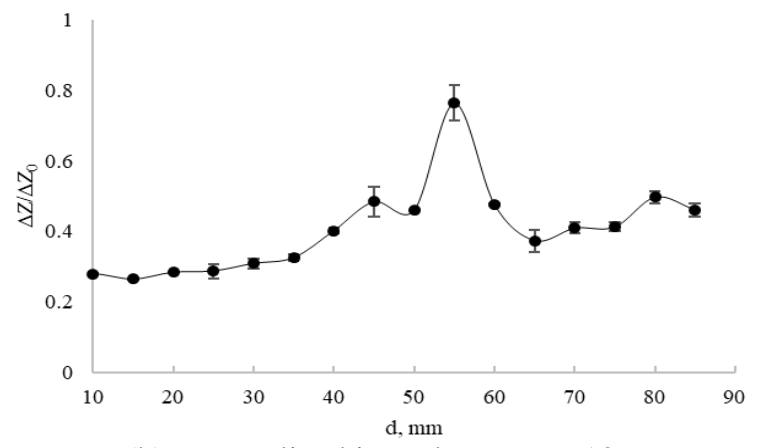

(b) Normalized impedance, $\mathrm{E}=10 \mathrm{~J}$

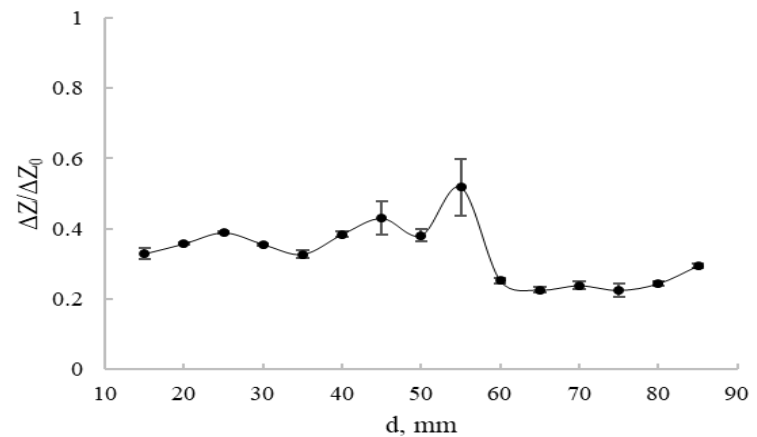

(d) Normalized impedance, $\mathrm{E}=20 \mathrm{~J}$

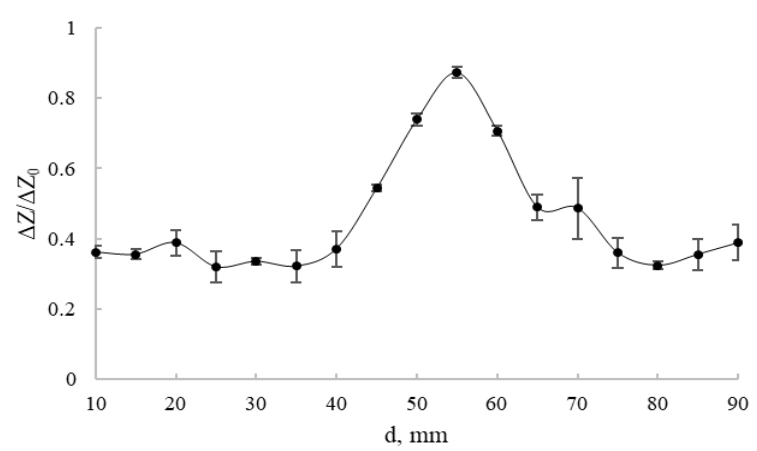

(f) Normalized impedance, $\mathrm{E}=30 \mathrm{~J}$

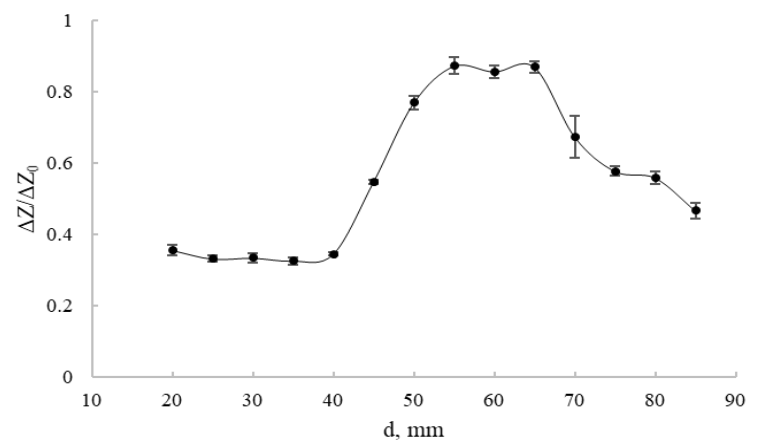

(h) Normalized impedance, $\mathrm{E}=30 \mathrm{~J}$

Fig. 8 Normalized measured impedance over damages by the impact; E: Energy of impact

Geometry of impact for the specimen no. 1 is shown in Fig. 8(a) where the normalized impedance increased up to 0.8 at the center of the impact which shows an increase up to $160 \%$ compared to the locations far from the impact's center, Fig. 8(b). Regarding the specimen no. 2 with the impact energy of 20 joules, the normalized impedance increased from 0.3 to 0.52 at the center of impact where the geometry and normalized impedance are shown in Fig. 8(c) and (d). Like the previous specimens, the peek normalized impedance increased from 0.35 to 0.87 , from 0.33 to 0.87 , from 0.24 to 0.96 and from 0.2 to 0.87 for specimens no.3, no. 4 , no. 5 and no. 6 , respectively. The increased amount was significant enough to be used as an indicator of damage due to the impact. Also, the approximate size of the damage can be estimated based on the graphs as well. For example, the visible length of damage for specimen no. 3 is approximately $45 \mathrm{~mm}$, and Fig. 8(f) shows that the normalized impedance increased in a range of almost $30 \mathrm{~mm}$, which is comparable with the actual length of the damage. 
However, as we discussed before, our proposed LAI technique is mainly a qualitative approach, and introducing a quantitative method was not the aim of this study. The sample impedance and frequency measurement over the surface of specimen no. 5 showed the approximate geometry of the damage where the normalized impedance increased over the damaged area, Fig. 7. The frequency shifting plot also detected the damage, but the plot was not as clear as the impedance measurements, and therefore, it was difficult to interpret the results in the case of a blind specimen evaluation.

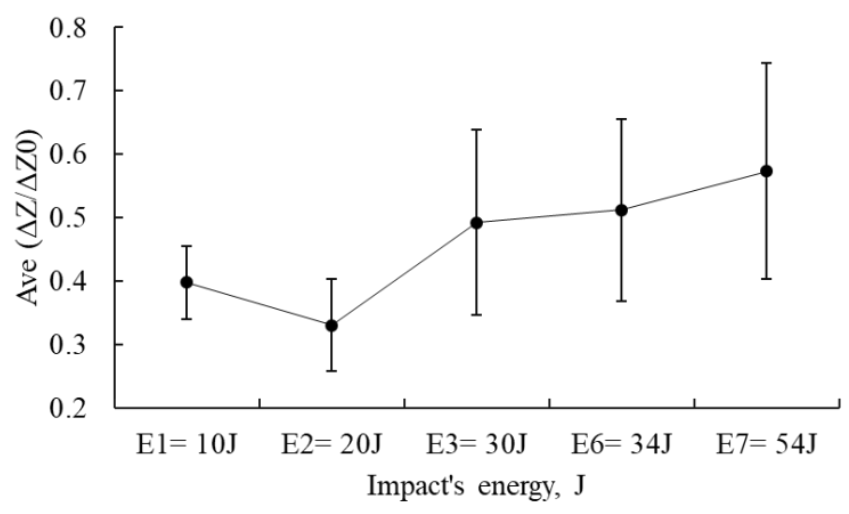

(a) Averaged normalized impedance versus impact energies

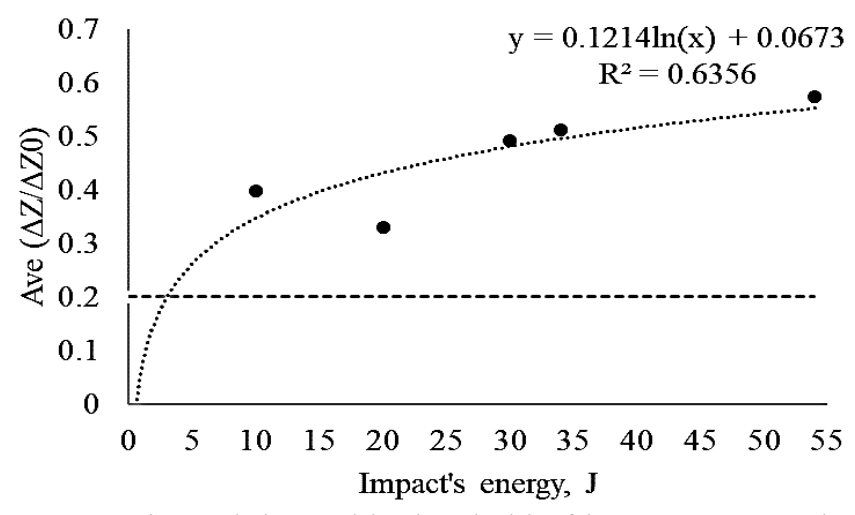

(b) An estimated detectable threshold of impact's energy by the proposed LAI setup

Fig. 9 Averaged measured impedance over middle and normal lines

The impedance results verified that the LAI technique could detect the impact's damage effectively. The normalized impedance increased over the region of the damage for an average of $100 \%$ compared to locations without any damage. This noticeable difference in measured impedance provides a reliable tool in qualitative and preliminary damage detection in composites. Also, the geometry of the damage can be estimated accurately. In Fig. 9(a), normalized impedances were averaged over the middle line, Ave $\left(\Delta \mathrm{Z} / \Delta \mathrm{Z}_{0}\right)$, and the standard deviations were calculated. This graph shows that the average normalized impedance increased from 0.3 to 0.6 when the energy of impact increased from 20 to 54 joules. We were also interested to know if we could estimate the minimum energy of impact detectable by the PZT transducer. On the other hand, we averaged the measured impedance over the surface of specimens far from the damage's region as an indicator of the healthy region. The baseline was found at 0.2 for the healthy regions. Then the best-fitted curve has been applied to the average of measured impedances. Since we were not sure which type of curve might fit the data points, we tested several curve fittings to have the best coefficient of determination $\left(\mathrm{R}^{2}\right)$ value and having zero impedance at zero impact energy as well. The final fitted curve was a logarithmic curve which crossed the 0.2 baseline at the impact's energy of 3 joules. Therefore, we assume that the 3 joules might be the minimum threshold of the energy that our setup can detect the damage for these specific specimens. The plot is shown in Fig. 9(b).

\section{Conclusions}

In this study, we introduced a novel, cost-effective, and efficient LAI technique. This technique could effectively detect the damage caused by impacts in fiberglass composite specimens. Briefly, the impedance signatures at different locations of the structure are measured, and the presence of damages are detected using their effect on the measured impedance.

First, we studied the effect of surface roughness on the measured impedance. It was shown that boundary conditions have a direct effect on the measured impedance magnitudes. The physical explanation of the soft and hard boundaries can be addressed using the system of springs and dampers, which has been discussed in earlier studies. As the main objective of this study, several FR4 fiberglass/epoxy composite specimens were tested using our proposed LAI setup. These specimens had damages caused by quantified and unquantified impacts. Different levels of impact energy were evaluated, and the setup could easily detect the damages for impact energies as low as 10 joules. The approximate geometry of the damages was also obtained. 
However, as we discussed, this technique is a qualitative method and can be used as a preliminary indicator of damages for further investigations.

The future work includes optimizing the current setup in terms of accuracy and data collection. The evaluation board could be more compact and assembled with the PZT transducer and its designed frame eventually. The current processing software can be extended to a smartphone application program with wireless communication with the transducer. Lastly, besides the SHM and NDT purposes, biomedical applications remain a very potential field for further investigation due to the LAI nature of biological structures, as well.

\section{Conflicts of Interest}

The authors declare no conflict of interest.

\section{Acknowledgment}

We would like to express our gratitude to Prof. Yaping Dan (UM-SJTU JI), Prof. Yu Zhefeng (School of Aeronautics and Astronautics, SJTU) and Prof. Aili Zhang (School of Biomedical Engineering, SJTU), Mr. Rui Zhang (UM-SJTU JI), Mr. Baojun Ning (School of Aeronautics and Astronautics, SJTU) and Mr. Sun Bin (Micro-Nano Key Institute, SJTU) for their support and contribution on designing the experiment setup and helping us navigate through the issues we encountered during the setup and experiments.

\section{References}

[1] S. Gholizadeh, "A review of non-destructive testing methods of composite materials," Procedia Structural Integrity, vol. 1, pp. 50-57, 2016.

[2] B. I. S. Murat, P. Fromme, M. Endrizzei, and A. Olivo, "Characterization of impact damage in composite plates," Strojniski Vestnik-Journal of Mechanical Engineering, vol. 64, no. 11, pp. 672-679, 2018.

[3] P. R. Monich, B. Henriques, A. P. Novaes de Oliveira, J. C. M. Souza, and M. C. Fredel, "Mechanical and biological behavior of biomedical PEEK matrix composites: A focused review,” Materials Letters, vol. 185, pp. 593-597, 2016.

[4] M. Naebe, M. M. Abolhasani, H. Khayyam, A. Amini, and B. Fox, "Crack damage in polymers and composites: a review," Polymer Reviews, vol. 56, no. 1, pp. 31-69, 2016.

[5] E. I. Croitoru, G. Oancea, and N. Constantin, "Impact testing on composite panels of fiberglass, carbon and Kevlar-carbon: a comparison and validation study," Materiale Plastice, vol. 54, no. 4, pp. 700-707, December 2017.

[6] S. Agrawal, K. K. Singh, and P. K. Sarkar, "Impact damage on fibre-reinforced polymer matrix composite - A review," Journal of Composite Materials, vol. 48, no. 3, pp. 317-332, 2013.

[7] A. Katunin, K. Dragan, and M. Dziendzikowski, "Damage identification in aircraft composite structures: A case study using various non-destructive testing techniques," Composite Structures, vol. 127, pp. 1-9, September 2015.

[8] H. Y. Nezhad, F. Merwick, R. M. Frizzell, and C. T. McCarthy, "Numerical analysis of low-velocity rigid-body impact response of composite panels," International Journal of Crashworthiness, vol. 20, no. 1, pp. 27-43, 2015.

[9] C. Maierhofer, P. Myrach, M. Reischel, H. Steinfurth, M. Röllig, and M. Kunert, "Characterizing damage in CFRP structures using flash thermography in reflection and transmission configurations," Composites Part B: Engineering, vol. 57, pp. 35-46, 2014.

[10] T. H. Loutas, A. Panopoulou, D. Roulias, and V. Kostopoulos, "Intelligent health monitoring of aerospace composite structures based on dynamic strain measurements," Expert Systems with Applications, vol. 39, no. 9, pp. 8412-8422, 2012.

[11] A. Ataş and C. Soutis, "Subcritical damage mechanisms of bolted joints in CFRP composite laminates," Composites Part B: Engineering, vol. 54, pp. 20-27, 2013.

[12] B. S. Divsholi and Y. W. Yang, "Combined embedded and surface-bonded piezoelectric transducers for monitoring of concrete structures,” Ndt \& E International, vol. 65, pp. 28-34, July 2014.

[13] W. S. Na and J. Baek, "A review of the piezoelectric electromechanical impedance based structural health monitoring technique for engineering structures," Sensors, Review vol. 18, no. 5, p. 18, May 2018. 
[14] F. G. Baptista, J. Vieira, and D. J. Inman, "Sizing PZT transducers in impedance-based structural health monitoring," IEEE Sensors Journal, vol. 11, no. 6, pp. 1405-1414, June 2011.

[15] S. Nezami, S. Lee, K. Kang, and J. Kim, "Improving durability of a vibration energy harvester using structural design optimization," no. 50497, p. V002T07A018, September 2016.

[16] H. Tinoco and D. Marulanda, "Damage identification in active plates with indices based on Gaussian confidence ellipses obtained of the electromechanical admittance," Journal of Nondestructive Evaluation, vol. 34, no. 3, article 28, 2015.

[17] K. S. Tan, N. Guo, B. S. Wong, and C. G. Tui, "Comparison of lamb waves and pulse echo in detection of near-surface defects in laminate plates,” NDT \& E International, vol. 28, no. 4, pp. 215-223, August 1995.

[18] E. La Malfa Ribolla, M. R. Hajidehi, P. Rizzo, G. F. Scimemi, A. Spada, and G. Giambanco, "Ultrasonic inspection for the detection of debonding in CFRP-reinforced concrete," Structure and Infrastructure Engineering, vol. 14, no. 6, pp. 807-816, 2018.

[19] R. Dugnani, "Novel transducer for characterization of low-impedance materials," Key Engineering Materials, vol. 558, pp. 435-444, June 2013.

[20] D. M. Ai, H. P. Zhu, H. Luo, and C. Wang, "Mechanical impedance based embedded piezoelectric transducer for reinforced concrete structural impact damage detection: a comparative study," Construction and Building Materials, vol. 165, pp. 472-483, March 2018.

[21] A. Narayanan, A. Kocherla, and K. V. L. Subramaniam, "Embedded PZT sensor for monitoring mechanical impedance of hydrating cementitious materials," Journal of Nondestructive Evaluation, vol. 36, no. 4, article 64, December 2017.

[22] H. A. Tinoco, L. Robledo-Callejas, D. J. Marulanda, and A. L. Serpa, "Damage detection in plates using the electromechanical impedance technique based on decoupled measurements of piezoelectric transducers," Journal of Sound and Vibration, vol. 384, pp. 146-162, December 2016.

[23] H. A. Tinoco, et al., "Evaluation of a piezo-actuated sensor for monitoring elastic variations of its support with impedance-based measurements," Sensors, vol. 19, no. 1, January 2019.

[24] R. Dugnani, "Novel nondestructive evaluation transducer for imaging of low-impedance targets," Journal of Intelligent Material Systems and Structures, vol. 26, no. 3, pp. 340-351, 2015.

[25] A. Mohammadabadi and R. Dugnani, "Damage detection in composites by LAI-PZT transducer," engrXiv, 2019.

[26] C. Liang, F. P. Sun, and C. A. Rogers, "Coupled electromechanical analysis of piezoelectric ceramic actuator-driven systems: determination of the actuator power consumption and system energy transfer," Proceedings of the SPIE Smart Structures and Materials Symposium, vol. 1917, pp. 286-298, 1993.

[27] A. Mohammadabadi and R. Dugnani, "Design and evaluation of a novel low acoustic impedance-based pzt transducer for detecting the near-surface defects," International Journal of Engineering and Technology Innovation, vol. 9, no. 3, pp. 196-211, March 2019.

[28] D. Benstock, F. Cegla, and M. Stone, "The influence of surface roughness on ultrasonic thickness measurements," The Journal of the Acoustical Society of America, vol. 136, no. 6, pp. 3028-3039, December 2014.

[29] J. A. Artero-Guerrero, J. Pernas-Sánchez, J. López-Puente, and D. Varas, "Experimental study of the impactor mass effect on the low velocity impact of carbon/epoxy woven laminates," Composite Structures, vol. 133, pp. 774-781, December 2015.

Copyright $(\mathrm{C}$ by the authors. Licensee TAETI, Taiwan. This article is an open access article distributed under the terms and conditions of the Creative Commons Attribution (CC BY-NC) license (https://creativecommons.org/licenses/by-nc/4.0/). 